\title{
Saline lavage and reaspiration for the diagnosis of periprosthetic joint infections
}

\author{
Hytham S. Salem ${ }^{1}$, Joseph O. Ehiorobo ${ }^{1}$, Kevin K. Mathew ${ }^{2}$, Michael A. Mont ${ }^{1}$ \\ ${ }^{1}$ Northwell Health Orthopaedics, Lenox Hill Hospital, New York, NY, USA; ${ }^{2}$ CUNY School of Medicine, New York, NY, USA \\ Correspondence to: Michael A. Mont, MD. Department of Orthopaedic Surgery, System Chief of Joint Reconstruction, Vice President, Strategic \\ Initiatives, Lenox Hill Hospital, Northwell Health, New York, NY, USA. Email: rhondamont@aol.com. \\ Provenance: This is an invited article commissioned by the Academic Editor Dr. Kai Chen (School of Biomedical Sciences, The University of Western \\ Australia, Perth, WA, Australia). \\ Comment on: Li R, Lu Q, Chai W, et al. Saline Solution Lavage and Reaspiration for Culture with a Blood Culture System Is a Feasible Method for \\ Diagnosing Periprosthetic Joint Infection in Patients with Insufficient Synovial Fluid. J Bone Joint Surg Am 2019;101:1004-9.
}

Submitted Nov 27, 2018. Accepted for publication Dec 13, 2019.

doi: $10.21037 /$ atm.2019.12.83

View this article at: http://dx.doi.org/10.21037/atm.2019.12.83

Periprosthetic joint infections (PJIs) are among the most dreadful complications associated with total joint arthroplasty (TJA). Despite the numerous precautionary measures employed by surgeons to avoid their occurrence, the incidence of PJIs after total knee arthroplasty (TKA) and total hip arthroplasty (THA) remains between $2.0 \%$ and $2.4 \%$ (1). Because the management of PJIs typically entails two-stage revision arthroplasty, it poses a substantial burden on patients, surgeons, hospitals, and healthcare in general. As the baby boomer generation continues to age, the incidence of TJA is increasing, and as a result, PJIs are expected to increase concordantly.

Early and accurate diagnosis of PJIs is of paramount importance to their successful management. Several synovial fluid and serum biomarkers have been identified that can help determine whether or not a PJI is present (2). While these tests are certainly useful clinically, they do not identify a specific organism to guide targeted antibiotic treatment. Therefore, synovial fluid culture remains critical for the work-up of patients with suspected PJI.

Sometimes, when attempting to aspirate synovial fluid for analysis, a dry tap precludes the ability to culture a sample. In fact, dry tap rates as high as $46 \%$ to $49 \%$ have been reported $(3,4)$. In these cases, saline can be injected into the joint and reaspirated in order to facilitate culture. However, there is a lack of consensus regarding the diagnostic accuracy of this technique. In fact, some authors do not believe that this technique can consistently yield fluid, or it may provide a sample that is not representative of native synovial fluid $(5,6)$. Conversely, other authors have reported that culture of saline injection and reaspiration is a sensitive and specific test that allows for susceptibility testing in the setting of a dry tap (3).

Li et al. (7) attempted to elucidate the feasibility of saline solution lavage and reaspiration for the diagnosis of PJIs in patients who have insufficient synovial joint fluid. A total of 286 hip or knee aspirations performed by a single surgeon were retrospectively reviewed. The indication for aspiration was any suspicion of a PJI including acute onset of pain, persistent pain since surgery, increased erythrocyte sedimentation rate (ESR) and/or C-reactive protein (CRP), or implant failure of unknown cause within 5 years of index surgery. If $\leq 1 \mathrm{~mL}$ of synovial fluid was aspirated, $10 \mathrm{~mL}$ of saline was injected and reaspirated, and the collected sample was divided into anaerobic and aerobic vials for culture. The samples were also evaluated using a leukocyte esterase strip test and sent to a laboratory for white blood cell and polymorphonuclear leukocyte evaluations. The presence of a PJI was determined by the modified Musculoskeletal Infection Society criteria (8). Direct aspiration was possible in 204 of the 286 cases $(71.3 \%)$ and the remaining 82 joints (28.7\%) underwent saline solution lavage. The diagnostic accuracy results of each cohort are presented in Table 1.

The results of this study indicate that saline solution lavage is feasible for the diagnosis of a PJI when synovial fluid cannot be aspirated. Authors that have recommended 
Table 1 Diagnostic accuracy

\begin{tabular}{lllll}
\hline Cohort & Sensitivity $(95 \% \mathrm{Cl})$ & Specificity $(95 \% \mathrm{Cl})$ & PPV $(95 \% \mathrm{Cl})$ & $\mathrm{NPV}(95 \% \mathrm{Cl})$ \\
\hline Overall $(\mathrm{n}=286)$ & $0.795(0.720-0.857)$ & $0.957(0.909-0.984)$ & $0.951(0.896-0.982)$ & $0.817(0.749-0.873)$ \\
Direct aspiration $(\mathrm{n}=204)$ & $0.768(0.672-0.847)$ & $0.991(0.948-1.000)$ & $0.987(0.930-1.000)$ & $0.819(0.741-0.882)$ \\
Saline lavage and reaspiration $(\mathrm{n}=82)$ & $0.851(0.717-0.938)$ & $0.857(0.697-0.952)$ & $0.889(0.760-0.963)$ & $0.811(0.648-0.920)$ \\
\hline
\end{tabular}

$\mathrm{Cl}$, confidence interval; NPV, negative predictive value; PPV, positive predictive value.

against this technique hypothesize that it yields diluted samples, thereby increasing the risk of false negative results. Interestingly, the sensitivity of saline solution lavage (85.1\%) was higher than that of direct aspiration (76.8\%), indicating a lower false negative rate in the saline lavage cohort. The other criticism of this technique is that the risk of contamination associated with saline injection may increase the rate of false positive results. The authors of this study noted 7 false positive results, 5 of which were determined to be due to coagulase-negative Staphylococcus infection. These results indicate that saline solution lavage may increase the risk of contamination and decrease the specificity of this diagnostic test.

The increased sensitivity and decreased specificity of reaspirated saline compared to direct aspiration are supported by the results of another study. Partridge et al. (3) reported that the sensitivity and specificity of the direct aspiration method were 81 and $90 \%$, respectively. In contrast, the saline injection and reaspiration method was $87 \%$ sensitive and $79 \%$ specific. Due to the emphasis that should be placed on ruling out suspected cases of PJI, improving its sensitivity at the expense of specificity may be an appropriate measure.

In both of these studies $(3,7)$, the saline lavage and reaspiration cohorts were comprised of patients from whom an adequate volume of synovial fluid could not be aspirated. This nonrandomized method of subject allocation may lead to an increased risk of selection bias. In order to combat this limitation, future studies should randomize patients into direct aspiration or saline lavage cohorts before aspiration is attempted. In this case, for patients who have adequate volumes of synovial fluid and are randomized into the saline lavage cohort, a direct comparison between the native joint fluid and the saline lavage-reaspiration samples can be carried out. A study conducted in this manner would help elucidate the true effect of saline lavage on joint fluid analyses.

Due to the limited availability of evidence to determine the utility of saline lavage and reaspiration for the diagnosis of PJIs, Li et al. (7) should be commended for their efforts in addressing this controversy. Randomized controlled trials are warranted to further clarify the effect of saline lavage on synovial fluid culture and other laboratory analyses.

\section{Acknowledgments}

None.

\section{Footnote}

Conflicts of Interest: The authors have no conflicts of interest to declare.

Ethical Statement: The authors are accountable for all aspects of the work in ensuring that questions related to the accuracy or integrity of any part of the work are appropriately investigated and resolved.

\section{References}

1. Kurtz SM, Lau E, Watson H, et al. Economic burden of periprosthetic joint infection in the United States. J Arthroplasty 2012;27:61-5.e1.

2. Saleh A, Ramanathan D, Siqueira MBP, et al. The Diagnostic Utility of Synovial Fluid Markers in Periprosthetic Joint Infection: A Systematic Review and Meta-analysis. J Am Acad Orthop Surg 2017;25:763-72.

3. Partridge DG, Winnard C, Townsend R, et al. Joint aspiration, including culture of reaspirated saline after a 'dry tap', is sensitive and specific for the diagnosis of hip and knee prosthetic joint infection. Bone Joint J 2018;100b:749-54.

4. Roberts P, Walters AJ, McMinn DJ. Diagnosing infection in hip replacements. The use of fine-needle aspiration and radiometric culture. J Bone Joint Surg Br 1992;74:265-9.

5. Lachiewicz PF, Rogers GD, Thomason HC. Aspiration of the hip joint before revision total hip arthroplasty. Clinical and laboratory factors influencing attainment of a positive 
culture. J Bone Joint Surg Am 1996;78:749-54.

6. Fehring TK, Cohen B. Aspiration as a guide to sepsis in revision total hip arthroplasty. J Arthroplasty 1996;11:543-7.

7. Li R, Lu Q, Chai W, et al. Saline Solution Lavage and Reaspiration for Culture with a Blood Culture System Is

Cite this article as: Salem HS, Ehiorobo JO, Mathew KK, Mont MA. Saline lavage and reaspiration for the diagnosis of periprosthetic joint infections. Ann Transl Med 2019;7(Suppl 8):S389. doi: 10.21037/atm.2019.12.83 a Feasible Method for Diagnosing Periprosthetic Joint Infection in Patients with Insufficient Synovial Fluid. J Bone Joint Surg Am 2019;101:1004-9.

8. Parvizi J, Gehrke T. Definition of periprosthetic joint infection. J Arthroplasty 2014;29:1331. 